\title{
Simultaneous Measurement of Temperature and Strain Based on Peak Power Changes and Wavelength Shift Using Only One Uniform Fiber Bragg Grating
}

abdollah malakzadeh ( $\sim$ afard77@gmail.com )

Institute for Research in Fundamental Sciences https://orcid.org/0000-0003-4864-2697

mohsen mansoursamaei

iran university of science and technology

Rasoul Pashaie

Iran university of science and technology

\section{Research Article}

Keywords: Fiber Bragg Grating, Strain Sensor, Temperature Sensor, FBG sensor, Cantilever Beam

Posted Date: March 15th, 2021

DOl: https://doi.org/10.21203/rs.3.rs-287636/v1

License: (c) (1) This work is licensed under a Creative Commons Attribution 4.0 International License.

Read Full License

Version of Record: A version of this preprint was published at Optical and Quantum Electronics on April 17th, 2021. See the published version at https://doi.org/10.1007/s11082-021-02865-4. 


\title{
Simultaneous Measurement of Temperature and Strain Based on Peak Power Changes and Wavelength Shift Using Only One Uniform Fiber Bragg Grating
}

\author{
Abdollah Malakzadeh ${ }^{1, *}$, Mohsen Mansoursamaei ${ }^{2}$, Rasoul Pashaie ${ }^{3}$ \\ ${ }^{1}$ School of Astronomy, Institute for Research in Fundamental Sciences, Tehran, Iran \\ ${ }^{2}$ Physics Department, EmamHossein University, Tehran, Iran \\ ${ }^{3}$ Physics Department, Iran University of Science and Technology, Tehran, Iran \\ E-mail: afard77@gmail.com
}

\begin{abstract}
Many efforts have been devoted to simultaneous measurements of strain and temperature by FBG sensors and several improving techniques have been resulted and implemented on the measurement. Most of them are based on two or more FBGs configurations or a single non-uniform FBG implementation. We propose simultaneous measurement of temperature and strain based on peak power changes and Bragg wavelength shifts using only one uniform fiber Bragg grating (FBG). We placed a ramp with the angle of $\theta$, similar to a tilted cantilever beam, on an assumptive structure and stuck a uniform FBG on it. When a uniform strain applied to a structure, the cantilever beam converts it to a non-uniform strain distribution along with itself and consequently the uniform FBG. By creating this non-uniform strain distribution, the peak power of the reflection spectrum of the FBG will be sensitive to strain changes. In addition, the Bragg wavelength shift will be sensitive to both temperature and strain parameters. According to our simulation, temperature sensitivity of $14.15 \mathrm{pm} /{ }^{\circ} \mathrm{C}$ is obtained for $\mathrm{FBG}$ sensor without any changes in the peak power. The strain sensitivity of $0.7837 \mathrm{pm} / \mu \varepsilon$, and a nonlinear sensitivity according to a quadratic function for peak power variation are also observed.
\end{abstract}

Keywords: Fiber Bragg Grating, Strain Sensor, Temperature Sensor, FBG sensor, Cantilever Beam

\section{Introduction}

optical fiber sensors, owing to their outstanding properties, have been widely developed for measuring temperature, strain, displacement, flow, vibration, bending, pressure, humidity, and etc. They are rapidly drawing interest in measurement of single, dual or multiple physical parameters [1-11]. Simultaneous measurement of temperature and strain is particularly important in many scientific and industrial applications and fiber Bragg grating (FBG) as a special class of optical fiber sensors has been successfully applied to these applications [12-13]. The FBG sensor is sensitive to both strain and temperature. However, it is impossible to discriminate between effects of strain and temperature by only reading Bragg wavelength shift.

Several techniques such as hybrid FBG/long-period grating (LPG) [14], different diameter FBG's [15], FBG in polymer optical fiber [16], FBGs written in fibers with different levels of doping elements [17] FBGs written in single mode fiber (SMF) and twin core fiber (TCF) [18], four-core fiber combined with a FBG [19], a combination of Fabry-Perot interferometer (FPI) and FBG [20], superstructure FBG [21], coated FBG [22], FBG in Polarization Maintaining Fiber 
(PMF) [23], $\pi$-Phase-Shifted FBG [24], chirped FBG [25], and FBG written in PANDA fiber [26] have been proposed to solve the problem. All works are also in the goal of minimizing the complexities as well as costs of structures of the sensors [27-28]. The techniques have mainly been based on detecting two physical indicators in reflection spectrum of FBG sensors that have different sensitivities to strain and temperature. Since there is only one physical indicator, Bragg wavelength shift, in a reflection spectrum of each uniform FBG, temperature and strain changes which both cause the Bragg wavelength shift, could not be identified by means of just one uniform FBG [29-30]. Thus, researchers mainly use more than one uniform FBG which causes several drawbacks including the complexities in the setup, overused of spectral sources and their costs. There are also innovative techniques using a single, but special FBG for the simultaneous measurement which have their own disadvantages such as: complexities in FBGs fabrication, and their difficult accessibility and costs.

In this article, we propose to use only one uniform FBG to measure simultaneously temperature and strain changes. In our design, an assumptive structure is affected by a uniform strain. A tilted cantilever beam, with a uniform FBG pasted on, is fixed to the structure to convert the uniformly applied strain to the structure into a non-uniform strain distribution along the cantilever. The uniform FBG would consequently experience non-uniform strain and show a sensitivity for the peak power in the reflection spectrum. Therefore, in addition to Bragg wavelength shift as the first indicator which is occurred due to temperature and/or strain changes, the peak power changes in the reflection spectrum of the uniform FBG is used as the second indicator for the simultaneous measurement of the temperature and strain changes.

\section{Measurement of temperature and strain}

According to the coupling-mode theory:

$\lambda_{\mathrm{B}}=2 \mathrm{n}_{\text {eff }} \Lambda$

where $\lambda_{B}$ is Bragg wavelength that depends on effective refractive index of fiber $\left(n_{\text {eff }}\right)$ and period

of grating $(\Lambda)$. When strain and temperature change, the wavelength shift of the peak wavelength is given as:

$$
\frac{\Delta \lambda_{\mathrm{B}}}{\lambda_{\mathrm{B}}}=(1-\mathrm{P}) \Delta \varepsilon+\left(\alpha_{\Lambda}+\alpha_{\mathrm{n}}\right) \Delta \mathrm{T}
$$

where $\Delta \varepsilon$ and $\Delta \mathrm{T}$ are the strain and temperature changes, respectively. For silica fiber, $\mathrm{P}=0.22$ is strain-optic coefficient, $\alpha_{\Lambda}=0.5 \times 10^{-6} / 0 \mathrm{~K}$ is thermal expansion coefficient and $\alpha_{\mathrm{n}}=6.9 \times 10^{-6} / 0 \mathrm{~K}$ is thermo-optic coefficient. Thus, reflection spectrum from the uniform FBG sensor is [31]:

$$
R(\lambda)=\exp \left[-\frac{4 \ln 2\left[\lambda-\left\{(1-P) \Delta \varepsilon+\left(\alpha_{\Lambda}+\alpha_{n}\right) \Delta T+1\right\} \lambda_{B}\right]^{2}}{c^{2}}\right]
$$

where $\mathrm{c}$ is light velocity. If there is no temperature change and only strain is changed, then, just the first part of the equation (2) will be used and if the strain is kept constant and temperature is 
changed, then, only the second part of the equation (2) will be activate. Fig. 1(a) and 1(b) show the FBG reflection spectra obtained at $200 \mu \varepsilon$ strain change with constant temperature value and $10^{\circ} \mathrm{C}$ temperature change with constant strain value at the Bragg wavelength of $1547 \mathrm{~nm}$, respectively. These diagrams are extracted by the conventional method of transfer matrix in FBGs and with the help of MATLAB software.
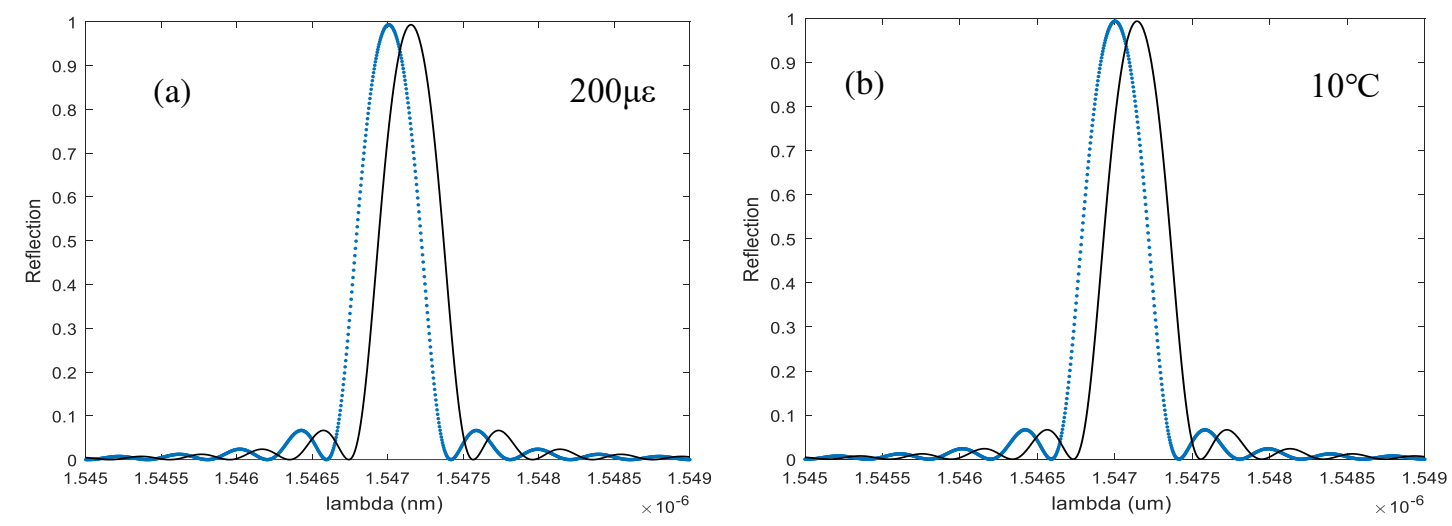

Figure (1): FBG reflection spectrum in $\lambda=1547 \mathrm{~nm}$, a) without external perturbations (point) and only the strain change by $200 \mu \varepsilon$ (line), b) without external perturbations (point) and only the temperature changes by $10^{\circ} \mathrm{C}$ (line).

According to figure (1), sensitivity of a uniform FBG to a uniform temperature change is 0.01415 $\mathrm{nm} /{ }^{\circ} \mathrm{C}$ and for uniform strain change can be obtained as $0.0012 \mathrm{~nm} / \mu \varepsilon$, at the Bragg wavelength of $1547 \mathrm{~nm}$ which are consistent with uniform FBG standard sensitivities [32].

\section{Measurement of strain in tilted cantilever beam}

A uniform strain is applied to the FBG resulting only a shift in Bragg wavelength. However, if a non-uniform strain distribution is created in the FBG, in addition to the Bragg wavelength shift, peak power of the reflection spectrum will also change [33]. As shown in Fig. 2, after applying the non-uniform strain distribution along a uniform FBG, the reflection spectra shift (Bragg shift) and their peak power undergo expansion.
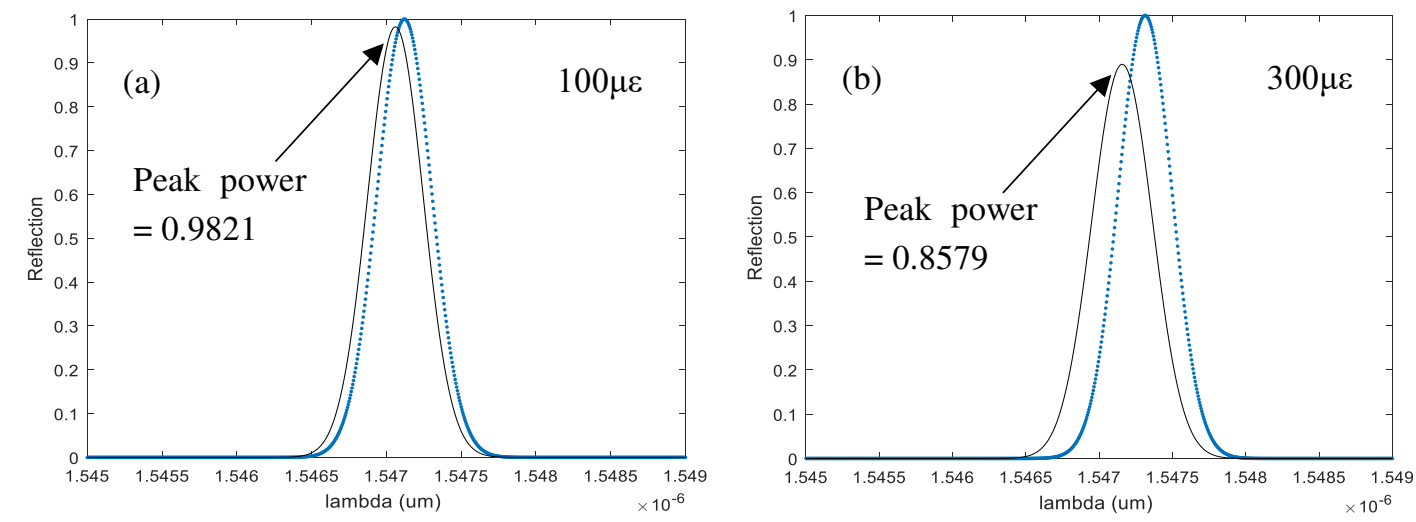

Figure (2): FBG reflection spectra for uniform (point) and non-uniform (line) strain distribution with the values a) $100 \mu \varepsilon$ and b) $300 \mu \varepsilon$ 
A cantilever is a rigid structural and mechanical element such as a beam or a plate, anchored at one end to a (usually vertical) support from which it protrudes. Strain distribution along the cantilever beam is non-uniform and linear [31]. In this study, a uniform FBG is pasted on a cantilever beam which is attached and fixed with a slight angle $\theta$ on an assumptive structure, as shown in Fig. 3.

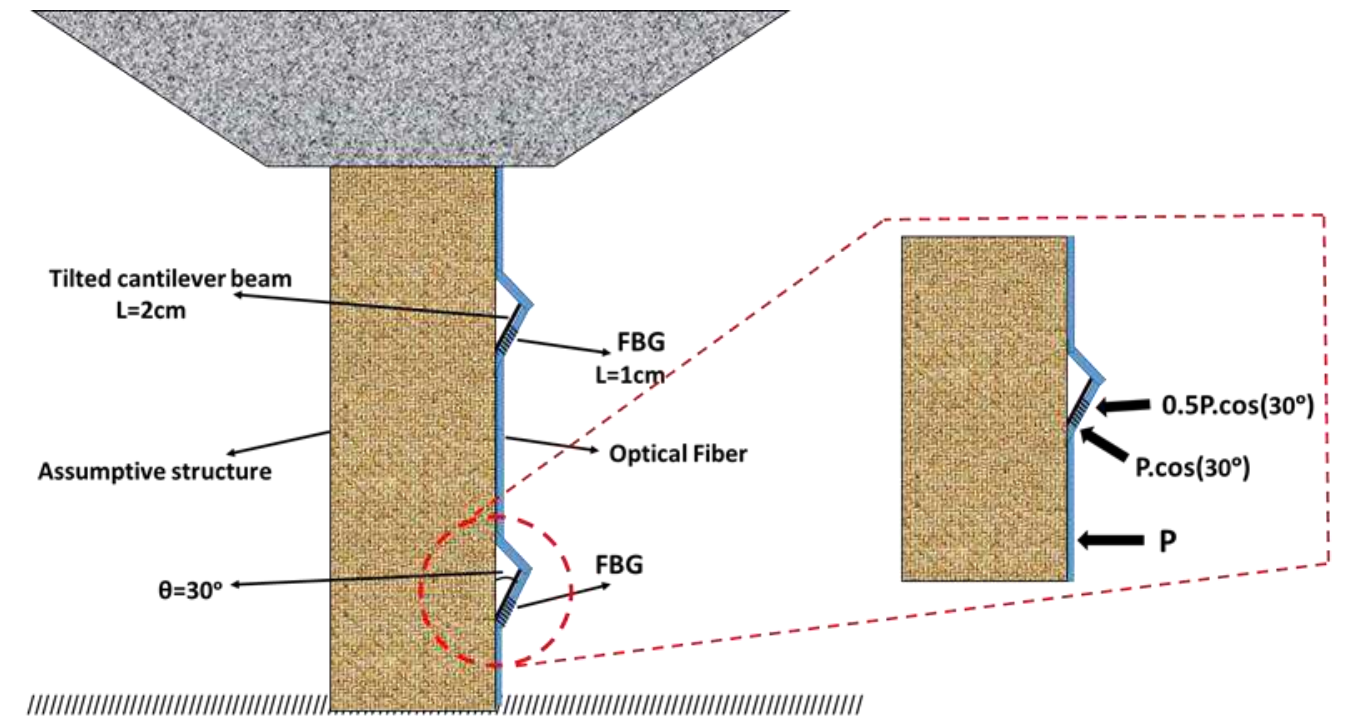

Figure (3): The mechanism of placing the tilted cantilever beam on the assumptive structure and the FBG on the tilted cantilever beam

There is a meaningful relation between strain applied to the structure $\left(\varepsilon_{\mathrm{s}}\right)$ and strain experienced at each part of the tilted cantilever beam $\left(\varepsilon_{c}\right)$, which depends on the inherent properties, thickness and length of the cantilever beam and the angle $\theta$. Parameter $\mathrm{M}$ is considered as an experimental strain coefficient to show this dependencies which can be between zero and one. The strain distribution in the cantilever beam is such that the fixed-end of cantilever suffers the most strain (M. $\left.\varepsilon_{s} \cdot \cos \theta\right)$ and decreases linearly to reach 0 at its free end.

Table 1: Maximum $\Delta \mathrm{P}$ according to the strain changes applied to the structure, assuming $\theta=30^{\circ}$ and $\mathrm{M}=1$.

\begin{tabular}{ccccc}
\hline $\begin{array}{c}\text { Strain in } \\
\text { structure } \\
\varepsilon_{\mathrm{s}}(\mu \varepsilon)\end{array}$ & $\begin{array}{c}\text { Maximum Strain in } \\
\text { start of FBG }(\mu \varepsilon) \\
\text { M. } \varepsilon_{s} \cdot \cos \theta\end{array}$ & $\begin{array}{c}\text { Maximum Strain in } \\
\text { end of FBG }(\mu \varepsilon) \\
1 / 2\left(\text { M. } \varepsilon_{s} \cdot \cos \theta\right)\end{array}$ & $\begin{array}{c}\text { Maximum } \\
\text { wavelength shift, } \\
1547 \mathrm{~nm}\end{array}$ & $\begin{array}{c}\text { Maximum } \\
\Delta \mathrm{P}=\mathrm{P}_{0}-\mathrm{P} \\
\mathrm{P}_{0}=1\end{array}$ \\
\hline 100 & 86.6 & 43.3 & 0.0783725 & 0.0034016 \\
200 & 173.2 & 86.6 & 0.156745 & 0.0134806 \\
300 & 259.8 & 129.9 & 0.2351175 & 0.02988734 \\
400 & 346.4 & 173.2 & 0.31349 & 0.05200299 \\
500 & 433 & 216.5 & 0.3918625 & 0.0791186 \\
600 & 519.6 & 259.8 & 0.470235 & 0.11034589 \\
700 & 606.2 & 303.1 & 0.5486075 & 0.1447415 \\
800 & 692.8 & 346.4 & 0.62698 & 0.18134794 \\
900 & 779.4 & 389.7 & 0.7053525 & 0.2192431 \\
1000 & 866 & 433 & 0.783725 & 0.257581678 \\
\hline
\end{tabular}


Depending on the position of the FBG on the cantilever beam, it can be seen that what percentage of strain applied to the assumptive structure reaches to the beginning of the FBG and also to the end of FBG. For example, if the length of FBG and tilted cantilever beam are $2 \mathrm{~cm}$ and $4 \mathrm{~cm}$, respectively, and the FBG is attached to the first half of the cantilever beam, a maximum strain equal to M. $\varepsilon_{s} \cdot \cos \theta$ is arrived at the beginning of the FBG and (1/2). (M. $\left.\varepsilon_{s} \cdot \cos \theta\right)$ is felt at the end of the FBG and the peak power will change with the strain applied to the structure shown in table 1. We assumed that $\theta=30^{\circ}$ and $M=1$ (meaning that all strain applied to the structure is transferred to the fixed-end of the tilted cantilever beam without any loss). As shown in table 1 the Maximum Bragg wavelength shift is linearly correlated with the applied strain on the structure with sensitivity equal to $0.7837 \mathrm{pm} / \mu \varepsilon$, while according to Fig. 4 , the relationship between the Maximum $\Delta \mathrm{P}$ and this strain is nonlinear.

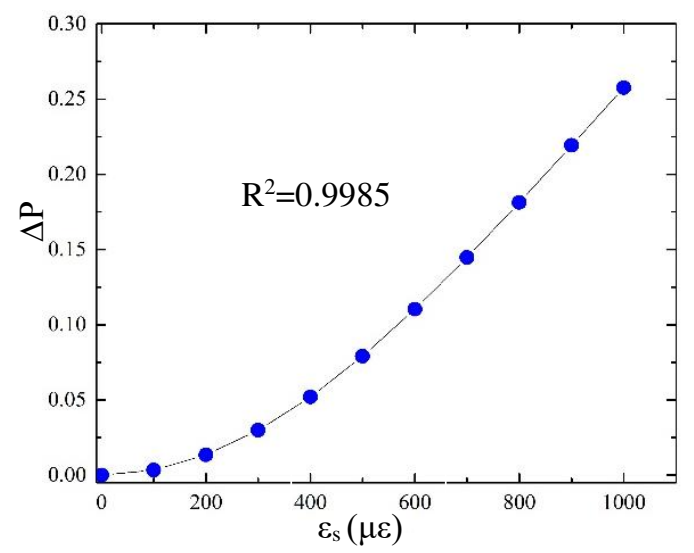

Figure (4): Relationship between $\Delta \mathrm{P}$ and the strain applied to the structure

Equation (4) shows the relationship between maximum $\Delta \mathrm{P}$ and the applied strain to the structure:

$$
\Delta \mathrm{P}=2 \times 10^{-7} \varepsilon_{\mathrm{s}}^{2}+7 \times 10^{-5} \varepsilon_{\mathrm{s}}-0.0048
$$

As can be seen from equation (4), by knowing the maximum peak power variations $(\Delta \mathrm{P})$, it is easy to obtain the strain applied to the structure without the need to know the other parameter such as temperature changes. For $\varepsilon_{\mathrm{s}}$ there are two values, one of which is discarded because it is a negative number and completely unrelated. Of course, we must note that equation (4) is a special equation for our example, and if the conditions and assumptions of our example (cantilever beam length, the cantilever angle $\theta$, FBG length and position on the cantilever) change.

\section{Simultaneous measurement of temperature and strain}

Simultaneous measurement of uniform temperature and strain changes on a structure has been topics of many researches. In this work, we introduce a tilted cantilever beam fixed on a structure, converting a uniformly applied strain to a structure into a non-uniform strain along the cantilever. A uniform FBG which is pasted on the cantilever beam would consequently experience a nonuniform strain and show a sensitivity to peak power value of the reflection spectrum. The strain changes in the structure, in addition to the Bragg wavelength shift $(0.0007837 \mathrm{~nm} / \mu \varepsilon)$, results in a 
nonlinear variation of peak power while the temperature changes only results in the Bragg wavelength shift $\left(0.01415 \mathrm{~nm} /{ }^{\circ} \mathrm{C}\right)$, because the temperature is applied uniformly distributed to the FBG. Thus, in an environment where both temperature and strain uniformly change, by measuring the maximum wavelength shift and minimum peak power value of the FBG reflection spectrum, both temperature and strain can be obtained by the following equations as:

$$
\begin{aligned}
& \Delta \mathrm{P}=2 \times 10^{-7} \varepsilon_{\mathrm{s}}^{2}+7 \times 10^{-5} \varepsilon_{\mathrm{s}}-0.0048 \\
& \Delta \lambda=0.000783725 \varepsilon_{\mathrm{s}}+0.01415 \Delta \mathrm{T}
\end{aligned}
$$

For example, Figure 5 shows a specific and uncertain environmental condition for our example (FBG is attached to the first half of the $4 \mathrm{~cm}$ cantilever beam), and we intend to obtain the temperature and strain changes applied to the structure.

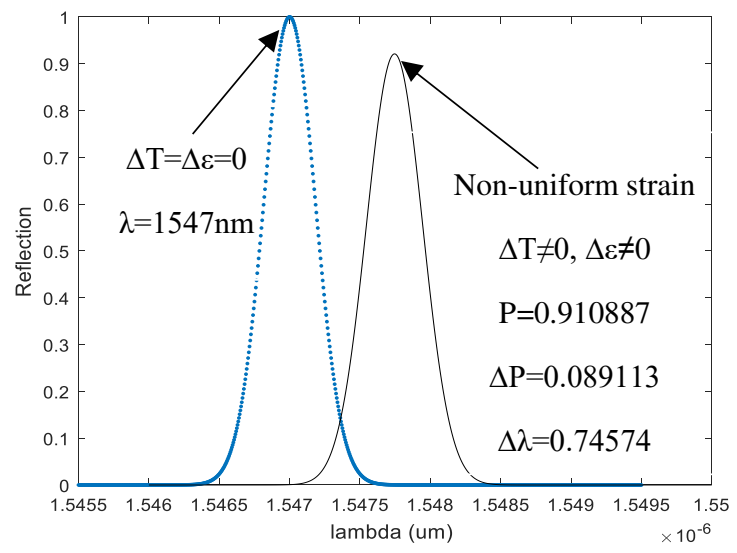

Figure (5): FBG reflection spectrum in constant strain and temperature (point) and in non-uniform strain and uniform temperature (line).

In this figure, the maximum $\Delta \mathrm{P}$ is equal to 0.08112 and maximum wavelength shift is 0.74574 . According to equation (5), the strain and temperature variations of structure are obtained equal to $503.34 \mu \varepsilon$ and $24.82^{\circ} \mathrm{C}$, respectively.

\section{Conclusion}

Many methods have been proposed for simultaneous measurement of temperature and strain by FBG sensors, although none of them can do this using only a single uniform FBG. Researchers have employed a variety of configurations including two or more FBGs, or one special FBG up to now. In this paper, we introduce a single uniform FBG pasted on a tilted cantilever beam which is fixed on a structure. This can be done with great precision. A uniform strain applied to the structure is distributed non-uniformly on a tilted cantilever beam and on the FBG. The non-uniform strain distribution on the FBG has led to a sensitivity of the peak power parameter to the strain. We exploit a non-uniform strain distribution along a uniform FBG concept resulting in changing in peak power of reflection spectrum in addition to the Bragg wavelength shift. Temperature and strain changes are obtained using Bragg wavelength shift and peak power changes equations. 
According to our results, temperature sensitivity of the FBG sensor to Bragg wavelength shift of $14.15 \mathrm{pm} /{ }^{\circ} \mathrm{C}$ and independent from peak power changes while the strain sensitivity of this sensor to Bragg wavelength shift is $0.7837 \mathrm{pm} / \mu \varepsilon$ and is connected to maximum peak power variation (or minimum peak power value) by a quadratic equation.

\section{Reference}

[1] Lu, Ping, et al, Distributed optical fiber sensing: Review and perspective, Applied Physics Reviews 6, 041302, (2019).

[2] Bao, Xiaoyi, and Liang Chen, Recent progress in distributed fiber optic sensors, Sensors 12, 8601-8639, (2012).

[3] Joe, Hang-Eun, et al, A review on optical fiber sensors for environmental monitoring, International journal of precision engineering and manufacturing-green technology 5, 173-191, (2018).

[4] A. Malakzadeh, M. Mansoursamaei, New matrix solution of the phase-correlation technique in a Brillouin dynamic grating sensor, J Optical Technology 85, 644-7, (2018).

[5] Sengupta, Somnath, Swapan Kumar Ghorai, and Palas Biswas, Design of superstructure fiber

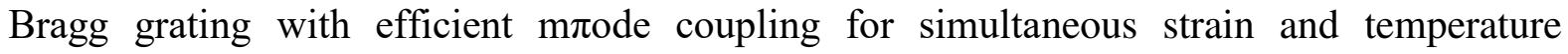
measurement with low cross-sensitivity, IEEE Sensors Journal 16, 7941-7949, (2016).

[6] A. Malakzadeh, M. Mansoursamaei and R. Pashaie, A novel technique in BDG sensors: combination of phase and frequency correlation techniques, Optical and Quantum Electronics 52, 1-10, (2020).

[7] Mihailov, Stephen J, Fiber Bragg grating sensors for harsh environments, Sensors 12, 18981918, (2012).

[8] A. Malakzadeh, R. Pashaie and M. Mansoursamaei, $150 \mathrm{~km} \varphi$-OTDR sensor based on erbium and Raman amplifiers, Optical and Quantum Electronics 52, 1-8, (2020).

[9] Lei, Xueqin, Xiaopeng Dong, and Chenxu Lu, Sensitive Humidity Sensor Based on a Special Dual-Mode Fiber, IEEE Sensors Journal 19, 2587-2591, (2018).

[10] A. Malakzadeh, R. Pashaie and M. Mansoursamaei, Gain and noise figure performance of an EDFA pumped at $980 \mathrm{~nm}$ or $1480 \mathrm{~nm}$ for DOFSs, Optical and Quantum Electronics 52, 1-16, (2020).

[11] A. Malakzadeh, M. Didar and M. Mansoursamaei, SNR enhancement of a Raman distributed temperature sensor using partial window-based non local means method, Optical and Quantum Electronics 53, 147, (2021).

[12] Ferreira, Luis A., et al, Simultaneous measurement of strain and temperature using interferometrically interrogated fiber Bragg grating sensors, Optical Engineering 39, 22262234, (2000).

[13] Su, Dan, et al, Compact Dual Fiber Bragg Gratings for Simultaneous Strain and HighTemperature Measurement, IEEE Sensors Journal 19, 5660-5664, (2019).

[14] Patrick, H. J., et al, Hybrid fiber Bragg grating/long period fiber grating sensor for strain/temperature discrimination, IEEE Photonics Technology Letters 8, 1223-1225, (1996). 
[15] Song, Minho, et al, Interferometric temperature-insensitive strain measurement with different-diameter fiber Bragg gratings, Optics letters, 22, 790-792, (1997).

[16] Qiu, Weiwei, et al, Simultaneous measurement of temperature and strain using a single Bragg grating in a few-mode polymer optical fiber, Journal of lightwave technology 31, 2419-2425, (2013).

[17] Iwashima, T., et al, Temperature compensation technique for fibre Bragg gratings using liquid crystalline polymer tubes, Electronics letters 33, 417-419, (1997).

[18] Kang, Zexin, et al, Twin-core fiber-based erbium-doped fiber laser sensor for decoupling measurement of temperature and strain, IEEE Sensors Journal 15, 6828-6832, (2015).

[19] Li, Chao, et al, Simultaneous measurement of refractive index, strain, and temperature based on a four-core fiber combined with a fiber Bragg grating, Optics \& Laser Technology, 90, 179184, (2017).

[20] Zhang, Xinpu, et al, Strain and temperature discrimination by using temperature-independent FPI and FBG," Sensors and Actuators A: Physical 272, 134-138, (2018).

[21] Guan, Bai-Ou, et al, Simultaneous strain and temperature measurement using a superstructure fiber Bragg grating, IEEE Photonics Technology Letters 12, 675-677, (2000).

[22] Sampath, Umesh, et al, Polymer-coated FBG sensor for simultaneous temperature and strain monitoring in composite materials under cryogenic conditions, Applied optics 57, 492-497, (2018).

[23] Uchimura, Ryotaro, et al, Fiber Fabry-Perot interferometric sensor using Bragg gratings in polarization maintaining fiber, Journal of Lightwave Technology 33, 2499-2503, (2014).

[24] Chen, Jiageng, Qingwen Liu, and Zuyuan He, High-Resolution Simultaneous Measurement of Strain and Temperature Using $\pi$-Phase-Shifted FBG in Polarization Maintaining Fiber, Journal of Lightwave Technology 35, 4838-4844, (2017).

[25] Liu, Weilin, Wangzhe Li, and Jianping Yao, Real-time interrogation of a linearly chirped fiber Bragg grating sensor for simultaneous measurement of strain and temperature, IEEE Photonics Technology Letters 23, 1340-1342, (2011).

[26] He, Xue Lan, et al, A Cascade Fiber Optic Sensors for Simultaneous Measurement of Strain and Temperature, IEEE Sensors Letters 3, 1-4, (2019).

[27] Liang, Minfu, Xinqiu Fang, and Yaosheng Ning, Temperature compensation fiber Bragg grating pressure sensor based on plane diaphragm, Photonic Sensors 8, 157-167, (2018).

[28] Guo, Kuikui, et al, Simultaneous Measurement of Strain and Temperature by a Sawtooth Stressor-Assisted Highly Birefringent Fiber Bragg Grating, Journal of Lightwave Technology 38, 2060-2066, (2020).

[29] Jiang, Nuan, et al, Simultaneous discrimination of strain and temperature using dual-gratings in one fiber, Optik 126, 3974-3977, (2015).

[30] Tao, Sicong,Xiaopeng Dong, and Bowen Lai, A sensor for simultaneous measurement of displacement and temperature based on the Fabry-Perot effect of a fiber Bragg grating, IEEE Sensors Journal 17, 261-266, (2016). 
[31] Mizutani, Y., and R. M. Groves, Multi-functional measurement using a single FBG sensor, Experimental mechanics 51, 1489-1498, (2011).

[32] Du, Jiangbing, and Zuyuan He, Sensitivity enhanced strain and temperature measurements based on FBG and frequency chirp magnification, Optics express 21, 27111-27118, (2013).

[33] Ling, Hang-yin, et al, Embedded fiber Bragg grating sensors for non-uniform strain sensing in composite structures, Measurement science and technology 16, 2415, (2005). 
Figures
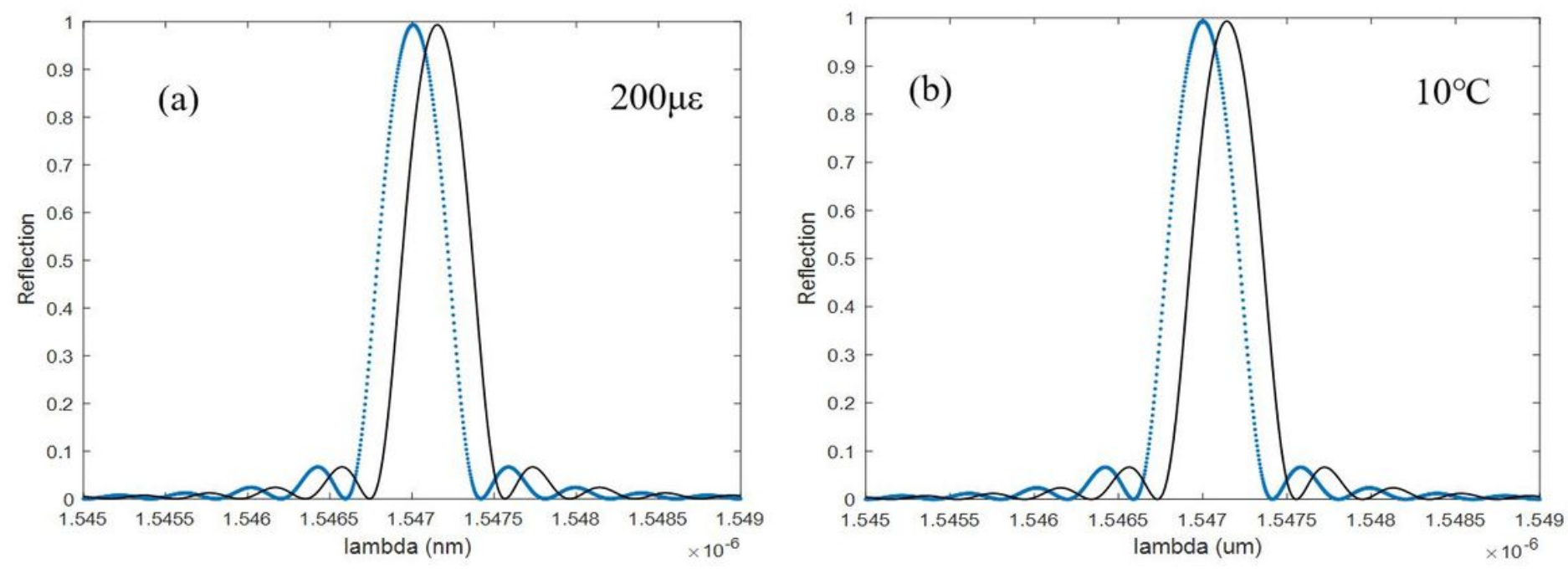

\section{Figure 1}

FBG reflection spectrum in $\lambda=1547 \mathrm{~nm}$, a) without external perturbations (point) and only the strain change by $200 \mu \varepsilon$ (line), b) without external perturbations (point) and only the temperature changes by 10区 (line).
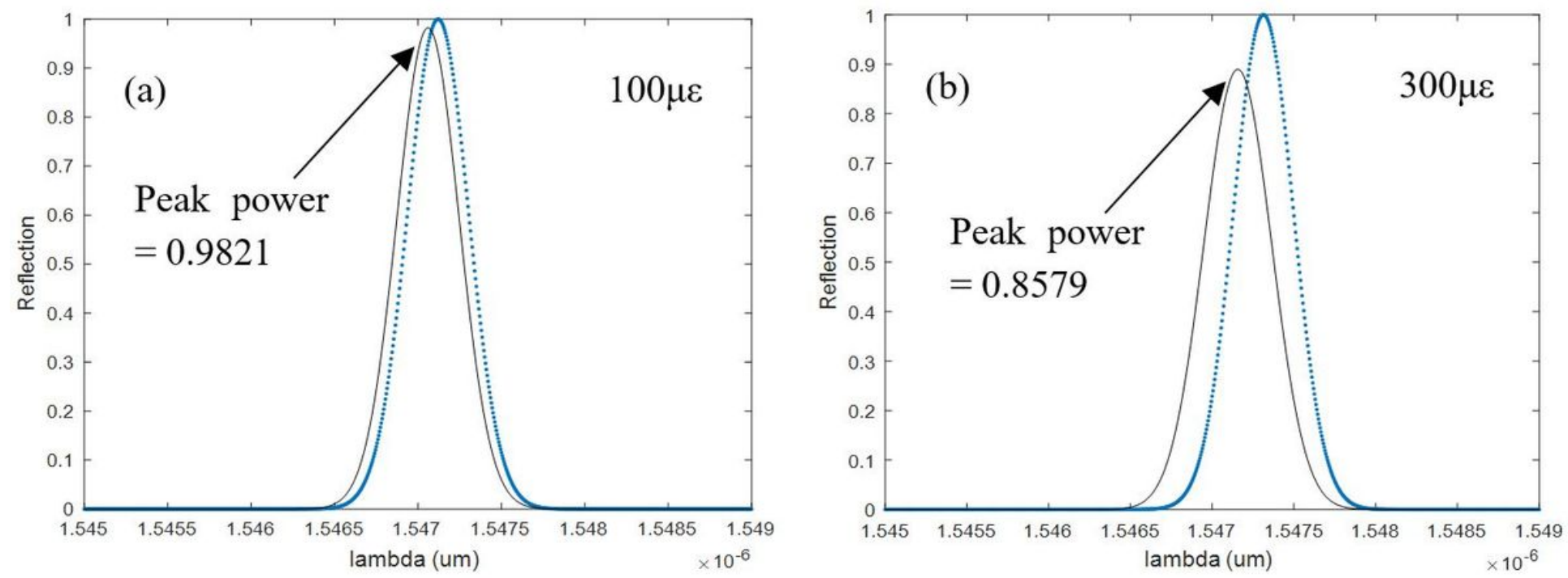

Figure 2

FBG reflection spectra for uniform (point) and non-uniform (line) strain distribution with the values a) $100 \mu \varepsilon$ and b) $300 \mu \varepsilon$ 


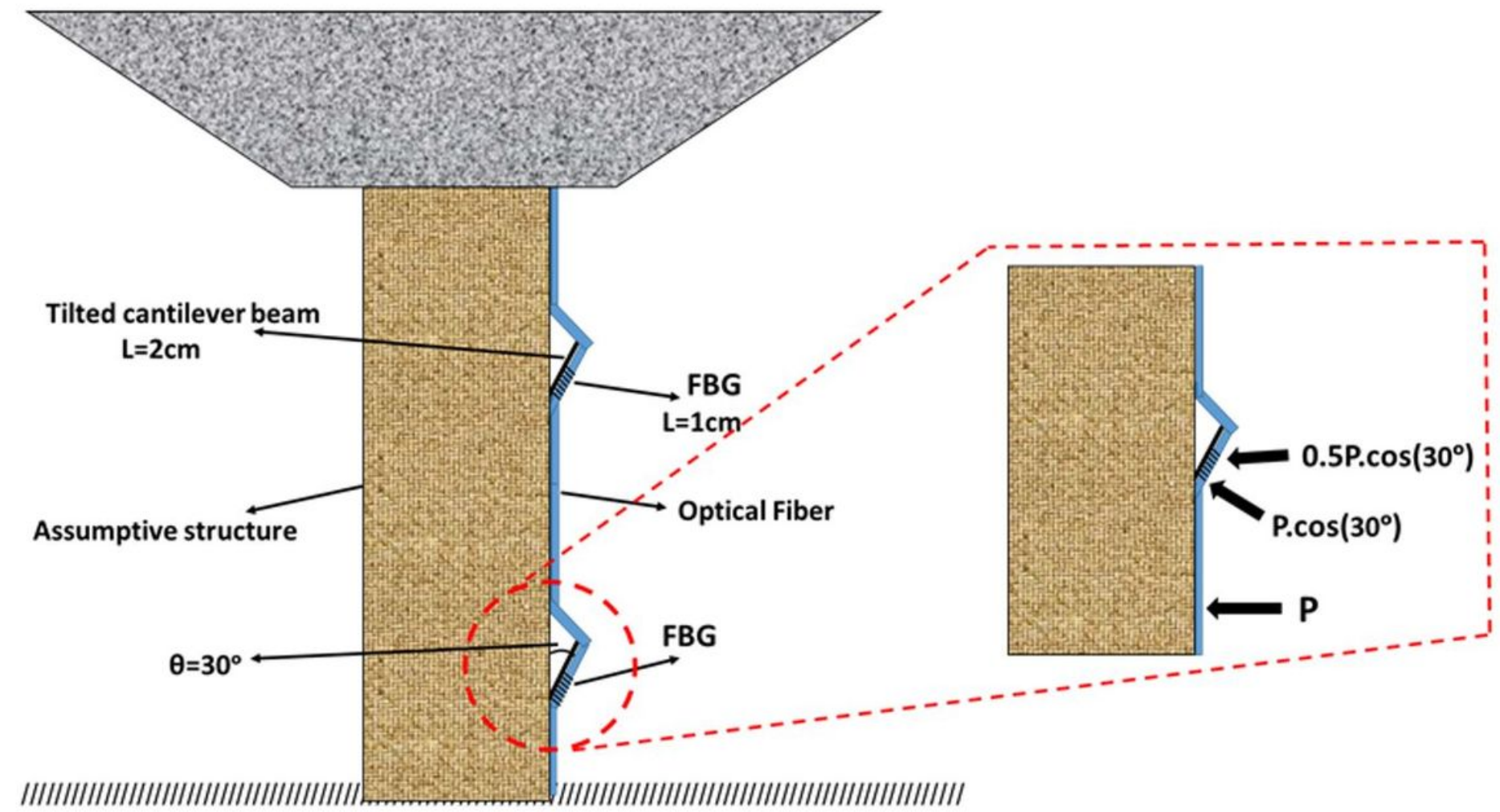

Figure 3

The mechanism of placing the tilted cantilever beam on the assumptive structure and the FBG on the tilted cantilever beam 


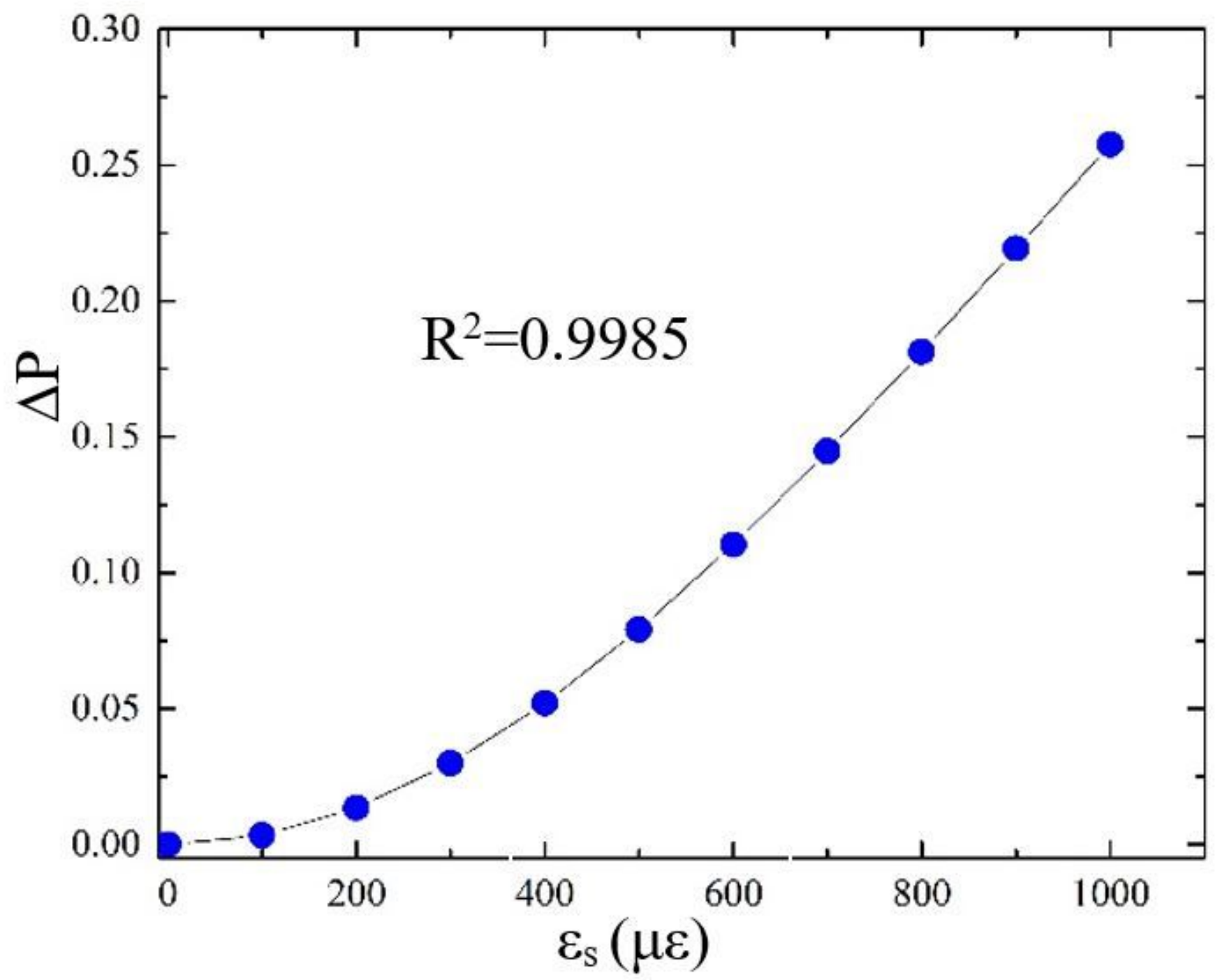

Figure 4

Relationship between $\Delta \mathrm{P}$ and the strain applied to the structure 


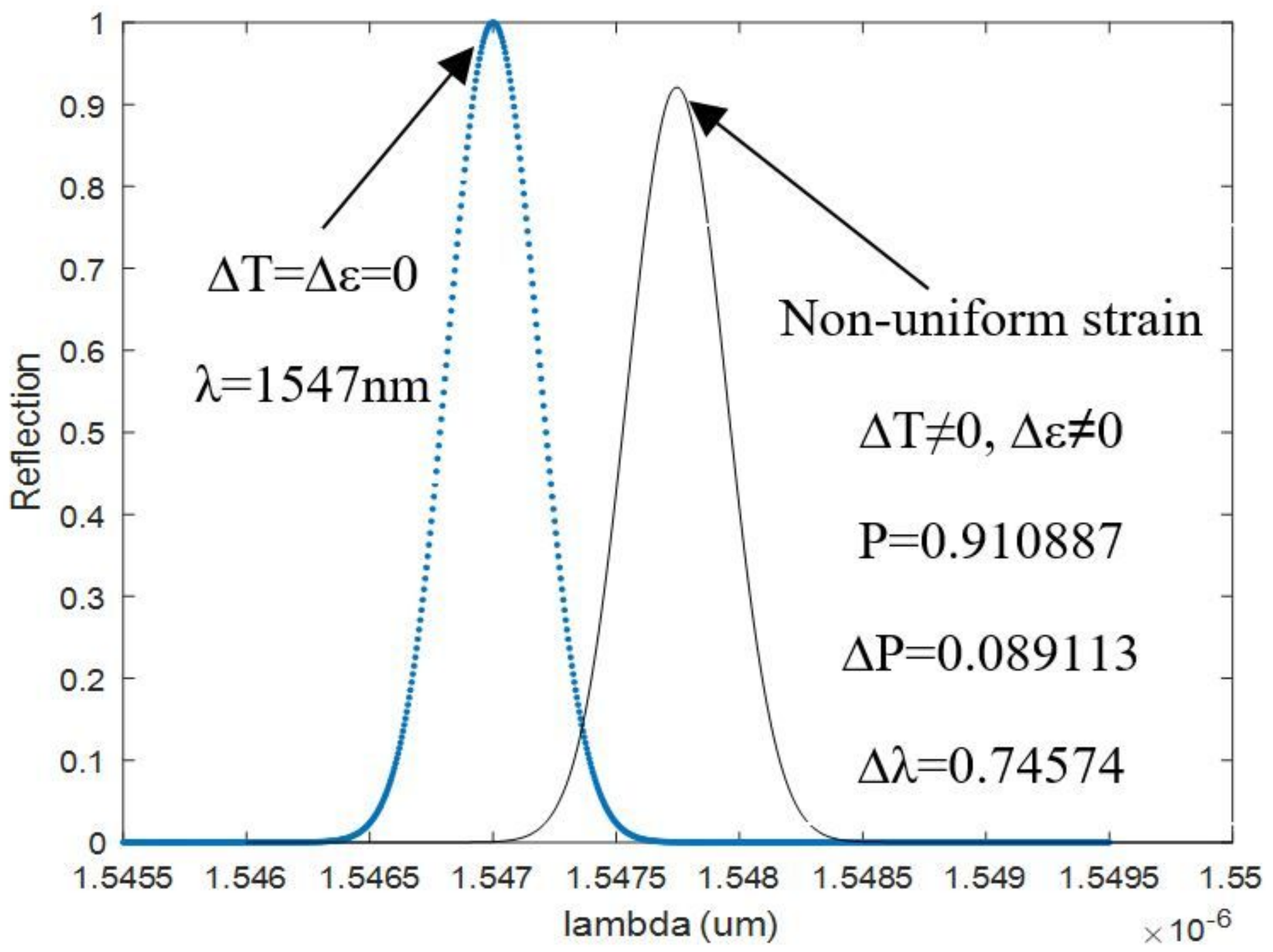

Figure 5

FBG reflection spectrum in constant strain and temperature (point) and in non-uniform strain and uniform temperature (line). 